\title{
Musculoskeletal effects of an electrical stimulation induced cycling programme in the spinal injured
}

\author{
K E Sloan, L A Bremner, J Byrne, R E Day, E R Scull \\ Department of Medical Physics, Royal Perth Hospital, Perth, Australia.
}

Twelve patients were involved in a 3 month stimulation induced cycling programme at the Royal Perth Rehabilitation Hospital. A number of the patients were less than 1 year post injury, all except one had an incomplete injury, and most were receiving physiotherapy. All patients who completed the programme increased their time of cycling and, in all but one case, the exercise load, indicative of a local training effect. Significant improvements were found in voluntary isometric strength, stimulated isometric strength and stimulated isometric endurance of the quadriceps, muscle grading of the quadriceps and biceps femoris and the cross-sectional areas of the quadriceps and total thigh muscle. No change was found in voluntary isokinetic strength of the quadriceps. All patients with incomplete injuries reported improvements in the activities of daily living (ADL) after the programme. Bone mineral density (BMD) was examined in two patients, one less than 1 year post injury, and one greater than 4 years post injury. The programme of cycling did not restore BMD in the latter patient. However, while the former patient still displayed a reduced BMD after the programme, it is unknown whether this loss of bone was retarded. This needs further investigation. This study demonstrates the effectiveness of a combined physiotherapy/cycling programme in the rehabilitation of people with spinal injuries. To be successful this type of programme has to be incorporated into the rehabilitation process, as has been done at the Sir George Bedbrook Spinal Unit.

Keywords: electrical stimulation; cycling; spinal injury; paraplegia.

\section{Introduction}

It has been shown that a spinal cord injury leads to secondary complications associated with inactivity. These complications include muscle atrophy, osteoporosis and cardiovascular deconditioning. However it has also been shown that regular exercise, produced either by voluntary activity or by electrical stimulation of paralysed muscles, can increase muscle strength and endurance in people with spinal injuries. ${ }^{1,2}$ An activity that combines the use of paralysed and voluntary muscle is stimulation induced cycling.

There have been many experimental exercise programmes conducted to examine the beneficial effects of stimulation induced cycling. However few, if any, have been incorporated into the rehabilitation regime of a recognised spinal unit or have attempted to apply the programme to patients less than 1 year post injury. This paper reports on a programme that attempts to do both.

\section{Methods}

\section{Patient selection}

Suitable spinal patients were referred to the functional electrical stimulation (FES) cycling programme by an orthopaedic consultant and the senior physiotherapist associated with the spinal unit at the Royal Perth Rehabilitation Hospital. Patients were at least 3 months post trauma, with either complete or incomplete upper motor neuron lesions between $\mathrm{C} 5$ and T12, and had upper body balance and some use of the 
upper limbs. Most patients were involved in a physiotherapy regime, in addition to participating in the cycling programme.

During the course of a 2 year period 12 patients participated in the programme (see Table I). Of these, nine remained in the programme for at least 3 months. Two of the patients who did not complete 3 months stopped because of increased spasm, whilst one patient withdrew for personal reasons.

\section{Cycling programme}

Prior to selection, trial stimulation of the quadriceps and hamstrings confirmed the presence of a useful muscle contraction. A trial session on the exercise bike determined the difficulty in transferring the patient to the bike, the strength of the stimulated muscle contraction and the ability of the patient to cycle without manual assistance. Other variables indicated by the trial session included the rapidity of muscle fatigue, the presence of muscle spasm and joint contractures, and the occurrence of adverse hyperreflexic symptoms. The presence of adverse symptoms excluded the patient from participation. All patients signed a consent form, which outlined the purpose and demands of the project.

Following assessment, patients participated in the cycling programme three times a week, for an initial 3 month period. The clinical exercise bike used for the programme has been described elsewhere. ${ }^{2}$ Each patient required an individual exercise protocol according to their muscle strength, level of spinal lesion and degree of completeness. For patients unable to maintain sustained exercise the cycling time was subdivided into three sets of 5 or 10 minutes, depending on the patient's current ability, separated by 5 minute rest periods. Cycling time was gradually increased for all patients to 30 minutes. Exercise load was increased in 5 Newton $(\mathrm{N})$ increments once the patient could confidently achieve 20 minutes of cycling without a load at 50-60 rpm for patients with incomplete injuries, and $30-40 \mathrm{rpm}$ for patients with complete injuries. Time and load were added according to the overload principle, keeping the speed unchanged. Fatigue was judged to have occurred if the cycle rate dropped by more than $10 \mathrm{rpm}$.

Heart rate was measured using a wristwatch style photo-optic pulse monitor at rest and at 5 minute intervals during cycling. Note was made of breathing rate, colour, sweating, dizziness and other hypertensive or adverse symptoms. Cycling was stopped immediately with the onset of adverse signs. Cycle time, rate and applied load were recorded for each session. The occurrence of functional and physiological changes were noted during the programme.

Table I Patient details

\begin{tabular}{ccccc}
\hline Patient & Sex & $\begin{array}{c}\text { Age } \\
\text { (yrs, months) }\end{array}$ & Level and lesion & $\begin{array}{c}\text { Post injury } \\
\text { (yrs, months) }\end{array}$ \\
\hline 1 & M & 28,2 & C5/6, IC & 11,6 \\
2 & M & 15,11 & T12, IC & 2,10 \\
3 & F & 19,7 & T4, C & 0,8 \\
4 & M & 33,10 & L1, IC & 0,11 \\
5 & M & 37,0 & T8/9, IC & 4,10 \\
6 & F & 39,8 & C7, IC & 0,8 \\
7 & F & 27,5 & C5, IC & 5,4 \\
8 & M & 54,8 & C6, IC & 4,5 \\
9 & F & 17,11 & T4/5, IC & 0,9 \\
10 & F & 19,7 & T3/4, IC & 0,2 \\
11 & M & 23,2 & T8, IC & 0,5 \\
12 & M & 17,4 & L1, IC & 0,3 \\
\hline
\end{tabular}

IC = incomplete lesion; $\mathrm{C}=$ complete lesion. 


\section{Measurements}

Several variables were measured at the beginning and at the end of a 3 month period, after at least 40 sessions on the bike (Table II). These included quadriceps strength and endurance, thigh muscle area, neurological muscle charts, bone density and a functional assessment.

Quadriceps strength, both isometric (where muscle tension develops but there is no change in length) and isokinetic (where tension developed by the muscle as it shortens is maximal at all joint angles over the full range of motion), and isometric endurance were measured using a Kincom isokinetic dynamometer (Chattecx Corp, Chattanooga, Tennessee, USA) (Fig 1). With the patient stabilised by pelvis belts, the rotational axis of the knee was aligned with the dynamometer. The lower leg was secured to the resistance pad without restricting ankle range of motion, approximately $2 \mathrm{~cm}$ above the ankle joint. The

Table II Measurement parameters used

\section{Measurement parameters}

1 Quadriceps cross-sectional area

2 Hamstrings cross-sectional area

3 Total midthigh muscle cross-sectional area

4 Neurological muscle grading

5 Quadriceps voluntary isometric strength

6 Quadriceps voluntary isokinetic strength

7 Quadriceps stimulated isometric strength

8 Quadriceps stimulated endurance

9 Bone densities of the femur and spine

10 Clinical and functional assessment

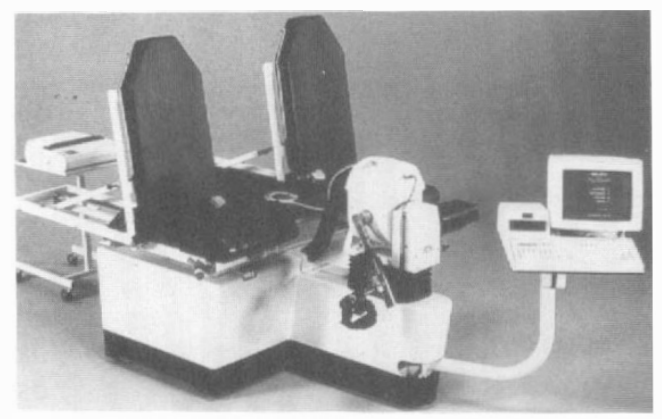

Figure 1 Kincom isokinetic dynamometer. moment arm was recorded as a reference for subsequent measurements. The weight of the leg was removed from all calculations, with the results expressed in Newton metres $(\mathrm{Nm})$.

A three electrode arrangement was used to stimulate the quadriceps muscle (Fig 2). The coordinates of the chosen electrode position were recorded for subsequent testing. Stimulation parameters used included a maximum current amplitude of 90 milliamps (the maximum amplitude of the commercially available Myocare stimulation units), a frequency of $25 \mathrm{~Hz}$ and a pulse duration of $300 \mu \mathrm{s}$.

Following three submaximal warmup contractions, the torque output of three maximum voluntary isometric contractions and three stimulation induced isometric contractions at $90 \%$ of the maximum current amplitude were recorded. This level of current was tolerable by all patients in the programme. Isometric tests were conducted at 60 degrees of knee flexion. One minute rest intervals separated the contractions, with 5 minutes between each set. Under isokinetic conditions, three voluntary concentric and eccentric quadriceps contractions at 20 degrees per second were measured. Peak torque output, average torque production across the full range of motion and torque at 60 degrees of flexion were recorded.

Muscle endurance was measured during a stimulation induced isometric contraction at $90 \%$ of the maximum current amplitude. The time taken for the contraction strength

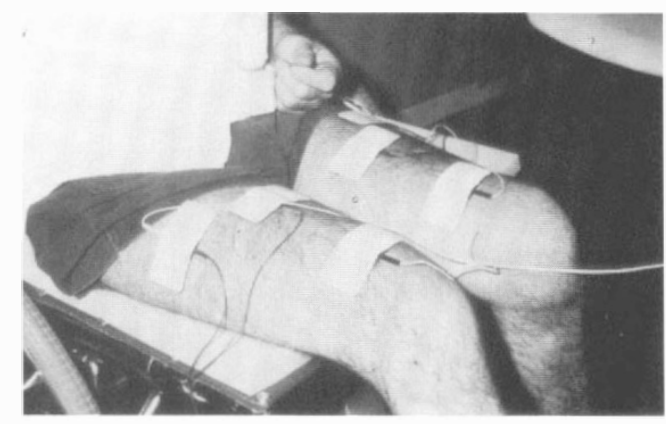

Figure 2 Three electrode arrangement to stimulate the quadriceps muscle. 
to drop to $50 \%$ of peak torque was recorded. Again the leg was in 60 degrees of knee flexion.

Strength and endurance measurements were conducted on both legs.

Thigh muscle area was measured using midfemoral computer tomography scans obtained from a Phillips Tomoscan CT scanner based at the Royal Perth Rehabilitation Hospital. A $5 \mathrm{~mm}$ thick scan slice was taken at the midpoint between the lateral tibiofemoral junction and the superior aspect of the greater trochanter. Scan time was 8 seconds with a magnification factor of 200 and scale factor of $50 \mathrm{~mm}$.

Scans were photographed to film and then imported into a Macintosh IIfx computer using a Ikegami CDD camera and RasterOps 364 video board. They were then analysed using a freely available image analysis program (NIH Image 1.4). Areas for the quadriceps, hamstrings, adductors, sartorius, and gracilis muscles and total muscle of the thigh were calculated. Confidence intervals (mean difference \pm 2 standard deviations (SD)) for the error due to repeating the measurement varied from $3.42 \mathrm{~cm}^{2}$ for the quadriceps to $0.66 \mathrm{~cm}^{2}$ for the hamstrings. Both legs were scanned in each patient.

Neurological muscle charts were performed by the supervising physiotherapist, noting motor deficit, graded voluntary muscle output, presence and subjective indication of degree of muscle spasticity and joint range of motion. The grades used to assess muscle strength were as follows.

0 No movement.

1 Flicker.

2 Movement of the limb segment through the full range without gravity.

3 Movement of the limb segment through the full range of movement against gravity.

4 Movement of the limb segment through the full range of movement against gravity and some resistance.

5 Normal movement and strength.

BMD was measured using dual energy $\mathrm{X}$ ray absorptiometry (DEXA) via a Hologic QDR 1000 machine (Fig 3). BMD of the spine from $\mathrm{L} 1$ to $\mathrm{L} 4$, the femoral neck,

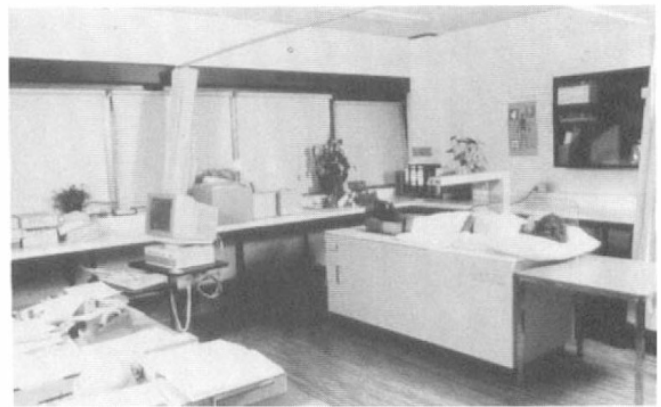

Figure 3 Hologic QDR 1000 machine.

trochanter, intertrochanteric line and Ward's triangle were measured for two patients, one who was in the programme for 1 year ( 8 months post injury) and one for 6 months (5 years post injury). Ward's triangle is the space formed by the angle of the trabeculae in the neck of the femur. Again both legs were measured.

A functional assessment of patient independence and ability with the activities of daily living was performed by an occupational therapist together with the subjective report from the patients themselves.

\section{Analysis}

Changes in the quadriceps endurance, muscle grading and thigh muscle area data after 3 months on the programme were analysed using dependent sample $t$ tests. The strength results, both stimulated and voluntary, did not conform to a normal distribution, showing signs of both skewness and kurtosis. Change in these variables was assessed using the Sign test, a nonparametric statistic for related samples which does not make assumptions about the form of the distribution. ${ }^{3}$ BMD changes were assessed using the $95 \%$ confidence limits for the difference between sequential readings on the same patient calculated by Haddaway and colleagues ${ }^{4}$ using DEXA.

\section{Results}

While efforts were made to involve patients who were between 3 and 12 months post injury, only four of the nine patients who cycled for 3 months fell into this category, 
and as such they have not been treated as a separate group.

All nine patients who completed 3 months on the programme increased their time of cycling, while eight of the nine patients also increased their cycling load. The average increase in time was 11.7 minutes while for load it was 30 newtons. The patient in whom load did not increase remained without a load throughout the 3 month programme. The speed of cycling remained relatively constant for all patients. Three patients who completed less than 3 months in the programme (5, 4 and 2 weeks respectively) also demonstrated increases in their cycling time and load applied. The changes in time and load are indicative of a local training effect.

A significant increase was seen for quadriceps cross-sectional area and total cross- sectional muscle area of the thigh (see Table III). No change was seen in the hamstring cross-sectional area after the programme.

Significant increases over the 3 month programme were found in voluntary isometric strength, stimulated isometric strength (Table IV) and stimulated endurance of the quadriceps (Table III). However isokinetic voluntary strength (Table IV) showed little change over the course of the programme.

Results of the muscle grading conformed with the isometric strength findings (Table III). Both the quadriceps and biceps femoris muscle gradings were significantly increased over the programme while the inner hamstrings showed no change.

BMD, measured in two subjects, showed no increase either in patient 6 who was on

Table III Changes in muscle area $\left(\mathrm{cm}^{2}\right)$, stimulated muscle endurance (s) and muscle grading (grade 1-5) assessed using dependent $t$-tests

\begin{tabular}{lccc}
\hline Variable & $\begin{array}{c}\text { Mean (SD) } \\
\text { at baseline }\end{array}$ & $\begin{array}{c}\text { Mean (SD) } \\
\text { at 3 months }\end{array}$ & Significance \\
\hline Quadriceps area & $48.1(13.8)$ & $52.6(12.1)$ & $p<0.002^{\mathrm{a}}$ \\
Hamstrings area & $21.0(8.6)$ & $21.3(8.0)$ & $\mathrm{NS}$ \\
Total area & $90.4(32.1)$ & $104.7(23.9)$ & $p<0.005^{\mathrm{a}}$ \\
Stimulated muscle & $24.2(20.7)$ & $42.4(22.9)$ & $p<0.014^{\mathrm{a}}$ \\
$\quad$ endurance & $3.2(1.4)$ & $3.7(1.3)$ & $p<0.047^{\mathrm{a}}$ \\
Quadriceps grade & $2.4(1.1)$ & $2.7(1.0)$ & $p<0.013^{\mathrm{a}}$ \\
Biceps femoris grade & $2.2(1.2)$ & $2.7(1.0)$ & $\mathrm{NS}$ \\
Inner hamstring grade & & & \\
\hline
\end{tabular}

aStatistically significant at $p<0.05$ level.

Table IV Changes in stimulated and voluntary isometric strength and voluntary isokinetic strength assessed using the sign test

\begin{tabular}{lc}
\hline Variable & Significance \\
\hline Voluntary isometric strength & $p<0.033^{\mathrm{a}}$ \\
Stimulated isometric strength & $p<0.011^{\mathrm{a}}$ \\
Isokinetic strength (concentric at 60 degrees) & $\mathrm{NS}$ \\
Isokinetic strength (concentric peak) & $\mathrm{NS}$ \\
Isokinetic strength (concentric averge) & $\mathrm{N}$ \\
Isokinetic strength (eccentric at 60 degrees) & $\mathrm{NS}$ \\
Isokinetic strength (eccentric peak) & $\mathrm{NS}$ \\
Isokinetic strength (eccentric average) & $\mathrm{a}$
\end{tabular}

aStatistically significant at the $p<0.05$ level 
the programme for 1 year (Table $\mathrm{V}$ ) or patient 8 who was on the programme for 6 months (Table VI).

Based on the reproducibility error reported, ${ }^{4} \mathrm{SC}$ showed significant decreases in the BMD of the femoral neck, trochanter, intertrochanteric line and Ward's triangle, in both legs. No change was found in the lumbar vertebrae. Both at the beginning and end of the programme the femoral neck BMD was below normal.

Patient 8, on the programme for 6 months, showed little change in all sites measured. Ward's triangle in both legs and the femoral trochanter in the left leg showed small but significant decreases. This patient's BMD at both the vertebral and femoral neck sites was substantially lower than normal and significantly lower than patient $6(p<0.03)$.

All incomplete patients subjectively reported improved function and wellbeing after the 3 month programme. Walking (with an aid), transferring, dressing and the tasks of daily living improved with the concomitant strength improvements. Change in spasm, characteristic of upper motor neuron lesions, was variable, although two patients had such large increases in spasm that they were forced to discontinue the programme. Of the patients who continued for 3 months, four had an increased frequency of spasm of a more severe nature, although it was still controllable. Three patients had no change, one felt there had been a decrease while one was unsure. Improvements were also noted in lower leg circulation, in terms of colour and temperature, especially in the patient with the complete lesion.

\section{Discussion}

Because of its clinical nature this study is unlike others reported in the literature. Patients were not obtained from outside the hospital for a specific project but were recruited while involved in an ongoing

Table $\mathbf{V}$ Changes in bone density $\left(\mathrm{g} / \mathrm{cm}^{2}\right)$ in patient 6 over the programme. Percentage of normal presented for lumbar veterbrae and femoral neck

\begin{tabular}{|c|c|c|c|c|c|c|}
\hline \multirow[t]{2}{*}{$\begin{array}{l}\text { Bone site } \\
\text { L1-L4 total }\end{array}$} & \multicolumn{2}{|c|}{$\begin{array}{c}\text { Baseline } \\
1.152(111 \%)\end{array}$} & \multicolumn{2}{|c|}{$\begin{array}{c}6 \text { months } \\
1.107(107.5 \%)\end{array}$} & \multicolumn{2}{|c|}{$\begin{array}{c}12 \text { months } \\
1.118(108.5 \%)\end{array}$} \\
\hline & Left & Right & Left & Right & Left & Right \\
\hline Femoral neck & $\begin{array}{c}0.787 \\
(92.6 \%)\end{array}$ & $\begin{array}{c}0.776 \\
(91.3 \%)\end{array}$ & $\begin{array}{l}0.71 \\
(83.5 \%)\end{array}$ & $\begin{array}{c}0.675 \\
(79.4 \%)\end{array}$ & $\begin{array}{c}0.625 \\
(75.5 \%)\end{array}$ & $\begin{array}{l}0.647 \\
(76 \%)\end{array}$ \\
\hline Femoral trochanter & 0.711 & 0.712 & 0.625 & 0.654 & 0.582 & 0.622 \\
\hline Femoral I/T line & 0.998 & 1.022 & 0.888 & 0.952 & 0.787 & 0.892 \\
\hline Ward's triangle & 0.69 & 0.68 & 0.571 & 0.596 & 0.503 & 0.587 \\
\hline
\end{tabular}

Table VI Changes in bone density $\left(\mathrm{g} / \mathrm{cm}^{2}\right)$ in patient 8 over the programme. Percentage of normal presented for lumbar veterbrae and femoral neck

\begin{tabular}{|c|c|c|c|c|}
\hline \multirow[t]{2}{*}{$\begin{array}{l}\text { Bone site } \\
\text { L1-L4 total }\end{array}$} & \multicolumn{2}{|c|}{$\begin{array}{c}\text { Base line } \\
0.795(76.4 \%)\end{array}$} & \multicolumn{2}{|c|}{$\begin{array}{c}6 \text { months } \\
0.819(78.8 \%)\end{array}$} \\
\hline & Left & Right & Left & Right \\
\hline Femoral neck & $\begin{array}{c}0.658 \\
(80.24 \%)\end{array}$ & $\begin{array}{c}0.675 \\
(82.32 \%)\end{array}$ & $\begin{array}{c}0.627 \\
(76.5 \%)\end{array}$ & $\begin{array}{c}0.638 \\
(77.8 \%)\end{array}$ \\
\hline Femoral trochanter & 0.497 & 0.485 & 0.468 & 0.475 \\
\hline Femoral I/T line & 0.894 & 0.869 & 0.88 & 0.856 \\
\hline Ward's triangle & 0.434 & 0.389 & 0.375 & 0.335 \\
\hline
\end{tabular}


rehabilitation programme. Therefore, it is difficult to separate the effects of the cycling programme from the effects of the physiotherapy regime. This applies especially to those patients who were less than 12 months post injury. Changes in the parameters measured should be seen as the combined effect of all treatment modalities employed at the Sir George Bedbrook Spinal Unit.

An increase in the exercise tolerance of all patients after 3 months in the programme was indicated by progressive increases in the treatment time and exercise load. This agrees with our previous finding ${ }^{2}$ and that of others. ${ }^{5-7}$

Exercise heart rate, although increased from resting levels, remained low in all patients, and local muscle fatigue was always the reason for discontinuing the session. This suggests a local (muscular) rather than central (cardiorespiratory) training effect. The lack of a central effect was reported by both Bremner et $a l^{2}$ and Regnarsson et al. ${ }^{6}$ It may indicate either a poor cardiac response due to decreased sympathetic effect or an inadequate intensity of work performed by weak muscles. ${ }^{6}$

It has been reported that when there is complete transection on the spinal cord above $\mathrm{T} 1$, the sympathetic influence on the heart is disrupted and the heart rate will not exceed $100 / 110 \mathrm{bpm} .{ }^{8}$ Phillips et al ${ }^{9}$ found no increase in heart rate during leg exercise in individuals with a complete lesion while the largest response was in the subject with the most incomplete injury. In a similar manner an increase in blood pressure was not found in complete paraplegics but was evident in incomplete paraplegics and tetraplegics. Ventilation and arterial blood gases also showed this variability. Other investigators, reporting similar findings, ${ }^{10,11}$ have concluded that the level and completeness of the injury are the important factors determining how normally a subject responds to exercise. In this study, while many of the patients had lesions above $\mathrm{T} 1$, all of these were incomplete, thus making it difficult to assess the sympathetic dysfunction. The one patient with a complete injury had the lesion at T4.

Many investigators have examined changes in thigh cross-sectional area after a programme of stimulation induced cycling, either using anthropometric methods or quantitative CT. Block et al, ${ }^{12}$ Pacy et al ${ }^{13}$ and Bremner et $a l^{2}$ all found significant increases in quadriceps area as measured by CT after a programme of cycling, while Ragnarsson et $a l^{6}$ and Phillips et al ${ }^{14}$ found increases in thigh girth, measured anthropometrically.

In this study both quadriceps and total muscle cross-sectional area were significantly increased, regardless of the initial strength of the patient or the completeness of the injury. The hamstrings remained unchanged, which may be due to the lower stimulation levels used in order to prevent spasm, or to the nature of cycling, which requires more work from the quadriceps than the hamstrings. ${ }^{15}$

The strength changes seen reflect those found by others. ${ }^{6,9}$ This study found significant increases in isometric voluntary strength, stimulated strength and stimulated endurance of the quadriceps muscles after a 3 month programme of stimulation induced cycling. These changes were seen, again, regardless of the initial strength of the patient. While most patients who had some voluntary movement were graded 3 or more by muscle charting there were two patients having grades less than 3 . These patients still exhibited improvements in all their strength measures. Difficulty was found in measuring patients with marginal strength using the Kincom isokinetic dynamometer, which seemed to lack the sensitivity required. One patient in particular was affected by this, and although classified incomplete with some residual movement in the quadriceps, was not included in the voluntary measurements.

The voluntary isokinetic strength results did not change significantly. over the programme, and this contradicts the other strength findings. The reason for this is not clear, although it may be related to the isokinetic contraction itself, which is unlike that performed in cycling, or to the spinal injury, which may restrict the development of maximum muscular tension over the full range of joint motion.

The muscle grading results compare favourably with the Kincom measurements. 
There was a significant increase in the quadriceps grade, matching the isometric strength findings. While biceps femoris also showed a significant increase the inner hamstrings did not, again highlighting the importance of the quadriceps, rather than the hamstrings, in cycling. ${ }^{15}$

BMD findings, that neither subject in which BMD was measured showed any increase, even after a year on the programme, agree with both Pacy et $a l^{13}$ and Leeds et al. ${ }^{16}$ Leeds et al found no change in BMD for the femoral neck, Ward's triangle or trochanter in six quadriplegic men after a 6 month programme or in three men after 3 years. All subjects were found to have osteoporotic proximal femurs when BMD was expressed as a percentage of their prematched controls. Pacy et al examined four paraplegic men after a programme of leg raises against a load using electrical stimulation followed by cycling induced by electrical stimulation. The total programme lasted for 42 weeks. These authors also found no change in either bone mineral content (BMC) or BMD during the exercise programme, even though all subjects had below normal femoral BMC and BMD.

It has been shown that immediately after a spinal cord injury increased calcium and hydroxyproline excretion is seen, indicating a loss of bone. ${ }^{17}$ There appears to be an increased but unbalanced rate of bone remodelling, with bone resorption occurring at a faster rate than bone formation, leading to osteoporosis. At approximately 2 years post injury a new equilibrium seems to be established between resorption and synthesis, although the bone mass remains at an 'osteoporotic' steady state. ${ }^{17,18}$

The decreases seen in patient 6 , only 8 months post injury, reflect this continuing imbalance between bone resorption and formation. Patient 8 , on the other hand, was at least 4 years post injury and while BMD was significantly below that of patient 6 it was relatively constant.

From this study, and from others already conducted, it appears that a programme of stimulation driven cycling does not increase BMD in the lower limb in patients 2 years post injury or longer. However, because no longitudinal studies have been conducted examining decreases in BMD in the spinal injured, it is difficult to know whether the cycling programme retarded loss of BMD in the patient less than 1 year post injury. This needs to be examined further.

The clinical findings reflect the significant improvements in strength and endurance of the lower limb muscles. Changes in ability to transfer, walk (with an aid) and in day to day tasks were found in all incomplete patients. The increases were assessed by an occupational therapist and subjectively by the patients themselves. This agrees with our previous findings. ${ }^{2}$ Spasm response to the programme seemed to vary greatly, with some patients having such a sudden increase that they were unable to continue. These patients, in general, had more spasm at the beginning of the programme than the other participants and this may reflect a spasm level that precludes inclusion in such a programme. More research needs to be conducted to confirm this. The patient with the complete injury, while not obtaining any functional improvement, reported improved skin health and temperature in the lower limbs. This has also been noted by Pacy et al. ${ }^{13}$

\section{Conclusions}

This study has demonstrated that a 3 month clinical programme of stimulation induced cycling, in conjunction with a normal physiotherapy regime, can produce significant increases in muscle strength, endurance and bulk. All patients involved displayed a training effect, cycling for longer and with a greater load.

Numbers did not allow the separation, for statistical purposes, of those patients less than 1 year post injury from those greater than 1 year. However improvements were seen regardless of the time elapsed since the spinal injury, which varied from 8 months to 5 years.

It is not known whether the introduction of such a programme shortly after a spinal injury will alleviate much of the accompanying muscle atrophy and loss of strength commonly seen. This needs further investigation. However a majority of the deconditioning occurs during the initial 6 months 
and it is therefore important to introduce therapy during this time. The cycling programme therefore has to become part of the rehabilitation process, as has been done successfully at the Sir George Bedbrook Spinal Unit.

This study was unable to prevent BMD loss or restore it after loss. However it is not clear if BMD loss is slowed by such a programme. Further studies need to examine the natural history of BMD loss and the effects of stimulation induced cycling on BMD in patients less than 2 years post injury.

\section{Acknowledgements}

The authors would like to acknowledge the great support provided by the orthopaedic and physiotherapy staff of the Sir George Bedbrook Spinal Unit and the enthusiasm and willingness of all participating patients.

\section{References}

1 Pollack SF, Axen K, Spielholz N et al (1989) Aerobic training effects of electrically induced lower extremity exercises in spinal cord injured people. Arch Phys Med Rehabil 70: 214-219.

2 Bremner LA, Sloan KE, Day RE, Scull ER (1992) A clinical exercise system for paraplegics using functional electrical stimulation. Paraplegia 30: 647-655.

3 Siegel S (1956) Nonparametric Statistics for the Behavioural Sciences. McGraw-hill Kogakusha, Ltd, Tokyo: $68-75$.

4 Haddaway MJ, Davie MW, McCall IW (1992) Bone mineral density in healthy women and reproducibility of measurements in spine and hip using dual energy X-ray absorptiometry. Br J Radiol 65: 213-217.

5 Petrofsky JS, Phillips CA (1984) The use of functional electrical stimulation for rehabilitation of spinal cord injured patients. Cent Nerv Syst Trauma 1: 57-73.

6 Ragnarsson KT, Pollack S, O’Daniel W et al (1988) Clinical evaluation of computerised functional electrical stimulation after spinal cord injury: A multicentre pilot study. Arch Phys Med Rehabil 69: 672-677.

7 Hooker SP, Figoni SF, Rodgers MM et al (1992) Physiologic effects of electrical stimulation leg cycle exercise training in spinal cord injured persons. Arch Phys Med Rehabil 73: 470-476.

8 Freyschuss U, Knutsson E (1969) Cardiovascular control in man with transverse cervical cord lesions. Life Sci 8: $421-424$.

9 Phillips CA, Petrofsky JS, Hendershot DM, Stafford D (1984) Functional electrical exercise: A comprehensive approach for physical conditioning of the spinal injured patient. Orthopaedics 7: 1112-1123.

10 Gruner JA, Glaser RM, Feinbeg SD et al (1983) A system for evaluation and exercise conditioning of paralysed leg muscles. J Rehab; Res Dev 20: 21-30.

11 Petrofsky JS, Phillips CA, Petrofsky SH (1985) Electronic physicians' prescription system for functional electrical stimulation patients. J Neurol Orthop Med Surg 6: 239-246.

12 Block JE, Steinbach LS, Friedlander AL et al (1989) Electrically stimulated muscle hypertrophy in paraplegia: Assessment by quantitative CT. J Comput Assist Tomogr 13: 852-854.

13 Pacy PJ, Halliday DA, Katz D et al (1988) Muscle and bone in paraplegic patients, and the effect of functional electrical stimulation. Clin Sci 75: 481-487.

14 Phillips CA, Danopulos D, Kezdi P, Hendershot D (1989) Muscular, respiratory and cardiovascular responses of quadriplegic persons to an FES bicycle ergometer conditioning program. Int J Rehabil Res 12: $147-157$.

15 Ericson M (1986) On the biomechanics of cycling: A study of joint and muscle load during exercise on the bicycle ergometer. Scand J Rehabil Med Suppl 16: 1-43.

16 Leeds EM, Klose J, Ganz W et al (1990) Bone mineral density after bicycle ergometry training. Arch Phys Med Rehabil 71: 207-209.

17 Chantraine A, Nusgens B, Lapiere ChM (1986) Bone remodelling during the development of osteoporosis in paraplegia. Calcif Tissue Int 38: 323-327.

18 Garland DE, Stewart CA, Adkins RH et al (1992) Osteoporosis after spinal cord injury. J Orthop Res 10: 371-378. 